\title{
Efficacy of L-Cysteine as an Anti-Oxidant in Papain Catalyzed Synthesis of Oligopeptides in Organic Solvent System
}

\author{
Santhana Srinivasan ${ }^{1,2,4 *}$, Shubhen Kapila ${ }^{1,3}$, Daniel Forciniti ${ }^{1,2}$ and Paul Nam ${ }^{1,3}$ \\ ${ }^{1}$ Center for Environmental Science and Technology, Missouri University of Science and Technology, Rolla, Missouri, USA \\ ${ }^{2}$ Department of Chemical and Biological Engineering, Missouri University of Science and Technology, Rolla, Missouri, USA \\ ${ }^{3}$ Department of Chemistry, Missouri University of Science and Technology, Rolla, Missouri, USA \\ ${ }^{4}$ Parenteral and Opthalmic Research and Development, Amneal Pharmaceuticals Pvt. Ltd. Ahmedabad, India
}

\begin{abstract}
Enzymatic peptide synthesis has drawn considerable attention for synthesis of high by-pass oligopeptide feed supplements in animal nutrition. A hardy protease, (papain) with a cysteine moiety at the active site requires the presence of an anti-oxidant in the reaction medium to ensure that the thiol group remains intact. Free cysteine has been the antioxidant of choice for papain-catalyzed synthesis of oligopeptides in aqueous systems. However, due to limited solubility of cysteine in organic solvents, it is generally not a suitable antioxidant for the synthesis of oligopeptides in biphasic solvent systems; instead, mercaptoethanol is often used. Lysine and Methionine is couple of well-known limiting amino acids that find application in cattle feed and poultry. Synthesis of co-oligopeptides of Lysine and Methionine has generally been attempted in bi-phasic solvent systems with Mercaptoethanol as an anti-oxidant. The inherent toxicity of mercaptoethanol when present even in trace amounts make its use undesirable during synthesis of oligopeptides to be used as feed supplements. Use of non-toxic antioxidants such as Cysteine would make the end product more amenable as a nutritional supplement for animal feed.
\end{abstract}

Therefore efficacy of L-cysteine as an anti-oxidant was investigated during papain catalyzed oligomerization of Lys, Arg, Glu and Asp in two organic systems: a three phase micro-aqueous media consisting of n-octane, DFP and water, as well as homogeneous ACN/water mixtures. Reactions were also carried out under an argon atmosphere in the presence and absence of anti-oxidants. The results of the experiments showed that L-cysteine facilitated oligomer synthesis in both the three phase system and the ACN/water mixture. The overall oligomer yields were found to be better than $75 \%$ in the presence of L-Cysteine. Oligopeptide yields obtained through reactions carried out under the argon atmosphere were less than $20 \%$.

Keywords: Lysine; Methionine; Arginine; Aspartate; Glutamate; Papain; L-Cysteine; Mercaptoethanol; Reverse-Phase Liquid Chromatography; Electro-spray ionization mass spectrometry

Abbreviations: Met: Methionine; Lys: Lysine; K: Lysine; M: Methionine; R: Arginine; LysEE: Lysine Ethyl Ester di-hydrochloride; MetEE: Methionine Ethyl Ester Hydrochloride; HPLC: High Pressure Liquid Chromatography; RPLC: Reverse Phase Liquid Chromatography; ESI-MS: Electrospray Ionization-Mass Spectrometry; HAS: Hexane sulfonic acid; UV/Vis: Ultraviolet/Visible; CAN: Acetonitrile; MS: Mass Spectrometry; MS/MS; Tandem Mass Spectrometry; Arg: Arginine; Asp- Aspartate; Glu: Glutamate; Cys: Cysteine; E: Glutamate; D: Aspartate; DFP: 1,1,1,2,3,4,4,5,5,5-decafluorpentane; DIPEA: N, N-diIsopropyl Ethyl Amine

\section{Introduction}

Primary function of proteolytic enzymes (proteases) is to catalyze hydrolysis of the peptide bond; however, under the right conditions these enzymes can also catalyze the synthesis of the peptide bond [1-5]. Papain belongs to a class of thiol proteases that have a cysteine residue at their active center. The activity of these proteases is based on a catalytic diad of cysteine and histidine [6,7].

Oligomers of Lys and Arg have potential application as high by-pass feed supplements in cattle-feed and poultry nutrition. Several studies have shown the optimal requirements of these amino acids in the diet and a complete discussion of the same is beyond the scope of this article and is available elsewhere [8-11]. Glu and Asp are di-carboxylic amino acids that are acidic at physiological $\mathrm{pH}$ [12]. Glu is one of the key molecules in cellular metabolism and can serve as metabolic fuel in the body when released as a consequence of hydrolysis of dietary proteins
[13-15]. It is present in high amounts in the blood and a small amount of it can easily permeate through the blood-brain barrier providing a fuel source for the brain [16]. Glu is also the most abundant excitatory neurotransmitter in the mammalian nervous system $[17,18]$. It helps to treat mental retardation, muscular dystrophy and ulcers also $[19,20]$. The sodium salt of Glu has a brothy taste at neutral $\mathrm{pH}$ and is used as a food additive to enhance the flavor of foods. Glu oligomers can be used in the fields of food and medicinal chemistry as a masking additive for bitter compounds in many foods [21]. These highly ionic peptides can also find applications as functional property modifiers. Asp also plays a crucial role in generating cellular energy [22,23]. Asp may provide resistance to fatigue and thus lead to endurance as a neuro-transmitter [24,25], although the evidence to support this idea is not strong. Asp is found in dairy, beef, poultry, sugar cane and molasses (the artificial sweetener aspartame is made from Asp and Phe). People with low proteinaceous diet or with eating disorders or malnutrition may develop an Asp deficiency and experience extreme fatigue or depression. Asp also helps in the removal of harmful ammonia from the body [26]. Glu

*Corresponding author: Srinivasan S, Center for Environmental Science and Technology, Missouri University of Science and Technology, Rolla, Missouri, USA, Tel: (95) 8511163560; E-mail: srivatin@gmail.com

Received: August 02, 2017; Accepted: September 19, 2017; Published September 28, 2017

Citation: Srinivasan S, Kapila S, Forciniti D, Nam P (2017) Efficacy of L-Cysteine as an Anti-Oxidant in Papain Catalyzed Synthesis of Oligopeptides in Organic Solvent System. Biochem Anal Biochem 6: 335. doi: 10.4172/2161-1009.1000335

Copyright: () 2017 Srinivasan S, et al. This is an open-access article distributed under the terms of the Creative Commons Attribution License, which permits unrestricted use, distribution, and reproduction in any medium, provided the original author and source are credited. 
and Asp oligomers have a potential use as slow release source in the food and medicinal chemistry [21]. Glu and Asp oligomers can serve as slow release source of Glu and Asp. Aqueous solvents are not suitable for enzymatic synthesis of these peptides because of the increased solubility of these hydrophilic oligomers in water [27].

Peptide synthesis catalyzed by cysteine proteases requires the presence of an antioxidant in the reaction medium to maintain enzymatic activity $[28,29]$. Free L-Cysteine has often been used as anti-oxidant during papain catalyzed oligopeptide synthesis in aqueous media; however, due to its limited solubility in organic solvents employed in the two phase systems during synthesis of polar amino acid oligopeptides mercaptoethanol has been used as antioxidant instead. However, mercaptoethanol is unsuitable for the synthesis of oligopeptides that are used in feed due to its toxicity. Mercaptoethanol is toxic (LD-50 $190 \mathrm{mg} \mathrm{kg}^{-1}$ in mice) and its presence in oligopeptides, even at trace level, can render oligopeptides unsuitable as feed supplements [30]. Hence, it is desirable to evaluate a non-toxic antioxidant in organic solvent systems for peptide synthesis.

Papain catalyzed synthesis of Leu, Met, Phe and Tyr oligomers in citric acid buffer in the presence of L-Cysteine has been reported, and oligomer yields range between $51 \%$ to $96 \%[31,32]$. It has been shown that papain can catalyze oligomerization of Met and Tyr in a monophasic system comprising of acetonitrile and citrate buffer with L-Cysteine as the reducing agent [33]. Papain catalyzed synthesis of a protected dipeptide BocGly-PheOMe in the presence of L-Cysteine has been carried out in an aqueous organic two-phase system consisting of different solvents like carbon tetrachloride, trichloroethylene, toluene and benzene [34]. Mercaptoethanol was used as an antioxidant in papain catalyzed hydrolysis and amino acid incorporation into BSA and Zein (a protein from corn) in low water organic media [35]. Mercaptoethanol has also been used for papain stabilization during the synthesis of LeuEnkaphalin precursors in ethyl acetate saturated with $\mathrm{Mes} / \mathrm{NaOH}$ buffer and Tris/HCl buffer [36,37]. Dithiothreithol has also been used as an anti-oxidant during papain catalyzed oligopeptide synthesis in water/water immiscible organic solvents such as hexane, toluene and chloroform [38]. We studied the use of L-Cysteine as a replacement for mercaptoethanol for papain catalyzed peptide synthesis in two organic solvent systems: A micro aqueous tri-phasic media and a monophasic media. Previous studies in tri-phasic media mainly reported the use of Mercaptoethanol as the anti-oxidant to obtain tailored short chain oligopeptides of lysine/ arginine with Methionine [27]. This study focused on the synthesis of only basic amino acid short chain peptides in tri-phasic solvent system. Animal studies of this peptide showed that the high toxicity of the resultant product rendered it ineffective for use as nutritional supplement. Rajesh et al. [28] have mainly studied the use of L-Cysteine as an anti-oxidant for the synthesis of oligopeptides of Methionine, phenylalanine and also HMB capped co-oligopeptides of Methionine and Phenylalanine in aqueous reaction systems $[39,40]$.

Basic and acidic amino acid oligomers find numerous applications as nutritional supplements and very limited studies have been conducted on the possibility of synthesizing such oligopeptides in either aqueous systems or in organic systems without Mercaptoethanol. The authors have already reported the potential of monophasic aqueous organic systems for the synthesis of oligopeptides of Lysine and Arginine [41]. This study utilized Mercaptoethanol as an antioxidant. Further animal studies showed that the product obtained from the synthesis with Mercaptoethanol was not suitable as a supplement for cattle feed due to its high toxicity. This necessitated an evaluation of other suitable anti-oxidants for synthesis of oligopeptides in both triphasic and monophasic aqueous organic reaction media.
The use of L-Cysteine as an anti-oxidant for peptide synthesis has been reported by numerous authors $[28,41]$. We decided to take advantage of the earlier reported studies to evaluate the efficacy of L-Cysteine in triphasic organic and monophasic aqueous organic reaction systems for protease catalyzed peptide synthesis.

Oligomers of Lys, Arg, Glu and Asp were synthesized in a threephase system consisting of n-octane, DFP and water and also in ACN/ water mixture. The efficacy of antioxidants (mercaptoethanol and L-Cysteine) and use of anoxic conditions on the oligopeptide yield and degree of oligomerization was evaluated. Concentration of L-Cysteine required for maintaining enzyme activity was optimized. The results obtained from this study were also compared with reported literature on the efficacy of L-Cysteine as antioxidant for protease catalyzed peptide synthesis.

\section{Materials and Methods}

\section{Materials}

L-Lysine ethyl ester (LysEE) dihydrochloride, L-Arginine ethyl ester (ArgEE) dihydrochloride, L-Aspartic acid (Asp), L-Glutamic acid (Glu), L-Cysteine hydrochloride monohydrate (Cys), n-Octane, 2-Mercaptoethanol, N, N diisopropylethylamine (DIPEA) and acetic acid were purchased from Sigma Chemical Co., (St. Louis, MO). Sodium salt of Hexane sulfonic acid (HSA), anhydrous ethanol (200 proof), O-Phosphoric acid, acetonitrile and ethanol (HPLC grade) were obtained from Fisher Scientific, (St.Louis, MO). 1,1,1,2,3,4,4,5,5,5-decafluorpentane (DFP) was purchased from MillerStephenson Chemical Company (Danbury, CT). Papain (EC 3.4.22.2, 25 units' activity $/ \mathrm{mg}, 28 \mathrm{mg}$ protein $/ \mathrm{mL}$ ) was provided by Novus International Inc., (St. Louis, MO). Argon (Grade 336) was purchased from Oz-Arc Gas, Rolla, MO. RPLC separation of oligomers was carried out with a XPERCHROM C-18 column $(250 \mathrm{~mm} \times 4.6 \mathrm{~mm})$ obtained from P.J. Cobert Associates Inc., (St. Louis, MO). Nanopure water used in the experiments was obtained after filtration through a Synergy 185 filtration system purchased from Millipore Corp. (Billerica, MA).

\section{LC with UV detection}

A model L-7000 HPLC system (Hitachi High Technologies America, San Jose, CA) was used to carry out the HPLC separations. The system consisted of a reciprocating piston pump (L-7100) fitted with a column oven (L-7300), autosampler (L-7200) and with a $50 \mu \mathrm{L}$ injection loop. The analytes separated on reverse phase columns were then introduced into a UV-Vis absorbance detector (L-7420).

\section{ESI-Mass spectrometer}

An Ion Trap Mass Spectrometer equipped with an Electrospray ionization interface (Model M-8000) purchased from Hitachi High Technologies America, San Jose, CA was used for the mass analysis of the synthesized oligomers.

\section{Synthesis of basic amino acid oligomers under anoxic condi- tions}

The synthesis of Lys and Arg oligomers was carried out in an anoxic condition using argon as the degassing agent. $25 \mathrm{~mL}$ of DFP, n-octane and water were taken in separate $100 \mathrm{~mL}$ round bottom flasks and purged with argon for 15 minutes. From these degassed solvents, 2.5 $\mathrm{mL}$ of DFP, $\mathrm{n}$-octane and $0.5 \mathrm{~mL}$ of water were added to another $25 \mathrm{~mL}$ round bottom flask that was already purged with argon for 15 minutes. To this, $123 \mathrm{mg}$ of L-LysEE was added. $100 \mu \mathrm{L}$ of degassed DIPEA was added to this reaction mixture. Purging was continued for another 15 
minutes. After purging, $30 \mathrm{mg}$ of papain was added and the reaction flask was sealed with stopper cock-Para film. The reaction media was incubated in a shaker for $24 \mathrm{~h}$. After $24 \mathrm{~h}$, the reaction was stopped by heating the contents at $80^{\circ} \mathrm{C}$ for 5 minutes. The reaction product was rotary evaporated to dryness. The resulting product was reconstituted in 50\% ethanol, diluted, centrifuged, filtered and injected into HPLC for product characterization. Yield was calculated based on the amount of residual monomer left in the reaction product after the incubation period. A similar procedure was used for Arg oligomerization except that $137 \mathrm{mg}$ of L-ArgEE was added as the substrate. A similar approach was used for the oligomerization of Arg and Lys in a $5 \mathrm{~mL} \mathrm{ACN} /$ water system consisting of $10 \%$ water. In this case, the supernatant and precipitate were separated, dried and reconstituted in 50\% ethanol/ water solution for HPLC characterization.

\section{Synthesis of basic amino acid oligomers in the presence of L- cysteine}

L-LysEE dihydrochloride $(123 \mathrm{mg}-0.5 \mathrm{mM})$ was added to a $7 \mathrm{~mL}$ clear glass reaction vial containing $2.5 \mathrm{~mL}$ of DFP, $2.5 \mathrm{~mL}$ of $\mathrm{n}$-octane and $0.5 \mathrm{~mL}$ of water. $100 \mu \mathrm{L}$ of DIPEA, $30 \mathrm{mg}$ of papain, and. $20 \mathrm{mg}$ of L-Cysteine were also added. The reaction vial was placed in an incubator shaker for a period of $24 \mathrm{~h}$. The reaction was stopped by heating the reaction mixture to $80^{\circ} \mathrm{C}$ for 5 minutes. The reaction product was rotary evaporated to dryness. The dried product was reconstituted in 50\% ethanol in water, centrifuged, filtered and analyzed in HPLC, to determine the amount of residual monomers and characterize the oligomers. Arg oligomerization was also carried out in the same procedure except that $0.5 \mathrm{mM}$ ArgEE was used as a substrate in that case. Lys and Arg oligomerization was also attempted in $5 \mathrm{~mL}$ of $\mathrm{ACN} /$ water mixture containing $10 \%$ water in the presence of L-Cys anti-oxidant. Other reaction conditions and additives were the same as used for three-phase system.

\section{Synthesis of Asp and Glu esters}

Asp and Glu were esterified with anhydrous ethanol in the presence of $\mathrm{HCl}$ gas using a procedure described by Rajesh et al. [28]. The synthesized ester was rotary evaporated to dryness. The dried ester was reconstituted in water, centrifuged, filtered and injected into RPLC for characterization. The percent conversion of monomers to ester was calculated based on the amount of residual monomers left in the product after the esterification reaction.

\section{Synthesis of acidic amino acid oligomers in organic solvent system}

Glu and Asp oligomers were synthesized from their corresponding esters in three-phase and monophasic solvent systems. The synthesis followed the same procedure as in Lys and Arg oligomerization. In this case, either $0.5 \mathrm{mM}$ Asp-diester or Glu-diester was added as the substrate. For oligomerization in monophasic system, $0.5 \mathrm{mM}$ Glu-diester/Aspdiester substrate was added to a $7 \mathrm{~mL}$ clear glass vial containing $0.5 \mathrm{~mL}$ of water and $4.5 \mathrm{~mL}$ of acetonitrile along with $100 \mu \mathrm{L}$ DIPEA and 30 $\mathrm{mg}$ of papain. The reaction mixture was incubated in a shaker for 24 h. After the incubation period, the mixture was heated at $80^{\circ} \mathrm{C}$ for five minutes to deactivate the enzyme. The supernatant was separated and rotary evaporated to dryness and the precipitate was lyophilized. The dried products were reconstituted in $50 \%$ ethanol, centrifuged, filtered and injected into HPLC for determining reaction yields based on the residual monomer. Product distribution information was obtained by injecting the dried precipitate into ESI (+)-MS. In both solvent systems, either $20 \mathrm{mg}$ of L-Cysteine or $25 \mu \mathrm{L}$ of 2 -mercaptoethanol was added as the anti-oxidant to maintain papain stability.

\section{HPLC analysis of synthesized oligomers}

The separation of residual Lys, Arg, Asp, Glu monomers and Lys, Arg oligomers was carried out with a reverse phase C-18 column (250 $\mathrm{mm} \times 4.6 \mathrm{~mm}$ i.d, $5 \mu$ ) and detected with a fixed wavelength UV-Vis detector maintained at $210 \mathrm{~nm}$ (Hitachi High Technologies, San Jose, CA). A gradient elution was used, the mobile phase gradient was changed from $100 \%$ A (Water $+10 \mathrm{mM}$ HSA $+0.1 \%$ O-Phosphoric acid) to $75 \% \mathrm{~B}(50 \%$ acetonitrile $+10 \mathrm{mM}$ HSA $+0.1 \%$ O-Phosphoric acid) in 50 minutes for the separation of residual monomers of Lys, Arg and the corresponding oligomers. In case of Asp and Glu oligomers, the separation of residual monomers was again carried out using a gradient elution with a mobile phase gradient from $100 \% \mathrm{~A}$ (Water + 5mM HSA $+0.1 \%$ O-Phosphoric acid) to $60 \% \mathrm{~B}(50 \%$ acetonitrile $+5 \mathrm{mM} \mathrm{HSA}$ $+0.1 \%$ O-Phosphoric acid) in 30 minutes. The mobile phase flow rate was maintained at $1 \mathrm{~mL} \mathrm{~min}{ }^{-1}$ and $10 \mu \mathrm{L}$ of the sample after filtration with a $0.22 \mu$ membrane filter was injected into the column in all the cases.

\section{ESI (+)-Mass Spectrometric characterization of acidic amino acid oligomers}

Asp and Glu oligomers were characterized by injecting them directly into an ESI-MS (M-8000, Model 3D-Q ion trap, Hitachi High Technologies, San Jose, CA). An electrospray ionization interface was used. The mass analyzer was scanned from $50-1200 \mathrm{amu}$. The operating parameters of the MS were as follows: Electrospray capillary voltage, $+3.5 \mathrm{KV}$; detector voltage, $400 \mathrm{~V}$; assistant gas heater temperature, $200^{\circ} \mathrm{C}$; desolvator temperature and the aperture- 1 temperature, $200^{\circ} \mathrm{C}$ and $150^{\circ} \mathrm{C}$ respectively. Asp and Glu oligomers were dissolved in ethanol/ water (50:50) mixture to form a nominal concentration of $0.5 \mathrm{mg} / \mathrm{mL}$ solution. The solution was injected into the MS using a syringe pump (Harvard Apparatus) at a flow rate of $1 \mathrm{~mL} / \mathrm{hr}$. A make-up solution ( $50 \%$ acetonitrile in water with $0.1 \%$ acetic acid) was infused along with the sample at a flow rate of $0.2 \mathrm{~mL} / \mathrm{min}$.

\section{Results and Discussion}

\section{Oligomer synthesis under an argon atmosphere}

The oligomerization of Lys and Arg in three-phase system was carried out under an argon atmosphere. The oligomerization was evaluated with and without antioxidants (L-Cysteine and mercaptoethanol). Oligomer yield was calculated on the basis of initial concentration of monomer (AA-EE) in the reaction and concentration of residual monomer (AA-EE + free AA) in the reaction mixture after the set incubation period.

$$
\begin{aligned}
& \text { Percent yield }=\left\{(\mathrm{AA}-\mathrm{EE})_{\text {initial }}-(\mathrm{AA}-\mathrm{EE}+\text { free } \mathrm{AA})_{\mathrm{final}}\right\} /(\mathrm{AA}-\mathrm{EE}) \\
& \text { initial } \times 100
\end{aligned}
$$

The residual amount of monomer and ester left in the reaction mixture was calculated based on the calibration curve obtained by determining the response of standards of the monomer and ester with HPLC-UV.

The RPLC separation of Lys oligomers synthesized under anoxic conditions with argon purging in three-phase system for a $24 \mathrm{~h}$ incubation period is shown in Figure 1. The chromatogram contains a series of peaks that appear after the Lys and LysEE peaks. Percent yield for [Lys] ${ }_{n}$ based on the formula mentioned above was determined to be approximately $30 \%$. Tentative identification of the oligomer peaks was based on ESI-MS results and expected increase in retention time with increased molecular weight within the homologous oligomers. 


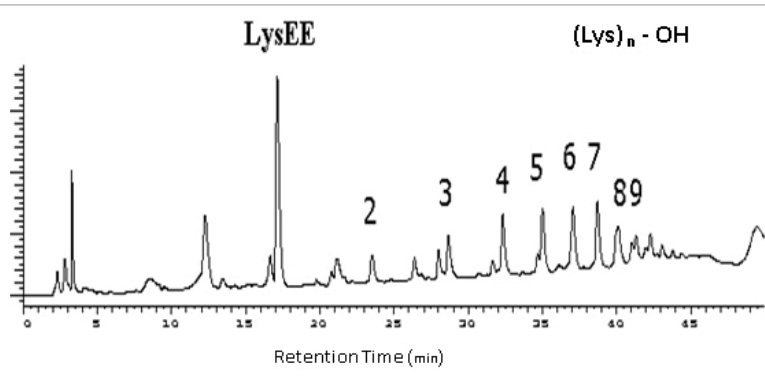

Figure 1: RPLC separation of Lys oligomers synthesized under anoxic conditions (argon atmosphere) in three-phase system. Separation was achieved with a RPLC C-18 column using a mobile phase gradient $100 \% \mathrm{~A}$ (Water $+10 \mathrm{mM} \mathrm{HSA}+0.1 \%$ O-Phosphoric acid) to $75 \%$ B (50\% acetonitrilze $+10 \mathrm{mM}$ HSA $+0.1 \%$ O-Phosphoric acid) in 50 minutes. The Chromatogram shows the presence of peaks corresponding to oligomers of Lysine.

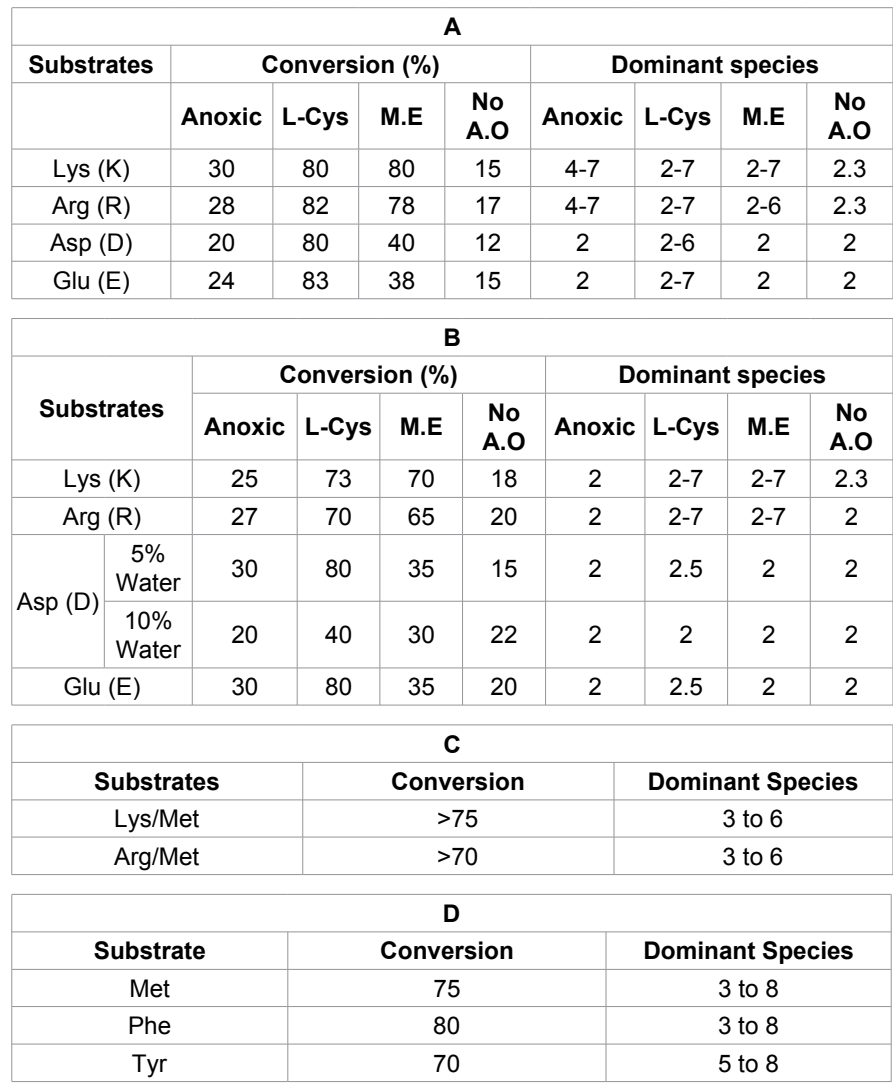

Table 1: Comparison of amino acid oligomerization efficiency of L-Cys mercaptoethanol and argon purge as anti-oxidant conditions for papain stabilization in (A) three-phase system and (B) monophasic (acetonitrile/water) system. (C) Amino acid oligomerization efficiency for basic amino acid oligomerization in three ph.ase system with Mercaptoethanol as antioxidant as reported by Roy et al. (D) Amino acid oligomerization efficiency with L-cysteine as antioxidant in aqueous system as reported by Rajesh et al. [28]

The degree of oligomerization ranged from 2-7 in case of [Lys] in three-phase system. Similar yield $(\sim 30 \%)$ was obtained for Arg oligomerization also in three-phase system. In this case, the degree of polymerization ranged from tetramer to heptamer. The percent yield for Lys oligomerization based on residual monomers left in the supernatant from a $10 \%$ water/90\% ACN mixture was determined to be $\sim 25 \%$ (Table 1 ).

The degree of oligomerization for Arg in case of a monophasic system ranged from 2-7. The results obtained for Arg oligomerization in
ACN/water system was similar to that obtained for Lys oligomerization (Table 1).

\section{Basic amino acid oligomerization in the presence of free cys- teine}

The synthesis of Lys oligomers in a three-phase system with free L-Cys as the anti-oxidant was also evaluated. The RPLC separation of Lys oligomers synthesized in three-phase system with L-Cysteine antioxidant for a $24 \mathrm{~h}$ incubation period is shown in Figure 2.

The oligomer yield was calculated on the basis of residual monomer found to be present in the reaction after the specific incubation period, as outlined earlier. The yield for [Lys] $]_{n}$ was found to be $\sim 80 \%$. The chromatogram consists of a series of peaks eluting after the retention time of LysEE. These peaks correspond to oligomers of Lysine. Tentative assignment of oligomer peaks was carried out on the basis of ESI-MS data and expected retention increase in a homologous series. Similar oligomer yield $(\sim 80 \%)$ was obtained in the case of $[\mathrm{Arg}]_{\mathrm{n}}$ in threephase system (Figure 3).

The Lys and Arg oligomers synthesized are mostly 2 to 7 residues long. The percent conversion for both Lys and Arg in 10\% water/90\% ACN mixture in the presence of L-Cysteine anti-oxidant was $~ 75 \%$ (Table 1). The degree of polymerization in this case ranged from 2-7 for both Lys and Arg. These results compare well with the yield and degree of polymerization obtained for basic amino acid oligomerization in both three-phase and monophasic systems with mercaptoethanol as the anti-oxidant $[28,33]$. The results in the presence of mercaptoethanol are also given in Table 1. With mercaptoethanol anti-oxidant, the yield in the three-phase system was close to $80 \%$ while it was close to $75 \%$ in case of ACN/water system [28,33]

This is significantly different from the results obtained in the absence of mercaptoethanol or L-Cysteine or argon purging (Table 1). In the latter case, the percent conversion was only $15 \%$ and the product mainly comprised of the dimer with very little trimer and tetramer.

These results confirm the need for an anti-oxidant in the reaction medium to maintain the activity of a thiol protease like papain. The anti-oxidants maintain the active site cysteine residue in its native thiol form and avoid the formation of disulfide bridges between the active site cysteine residue with another cysteine residue (Cys-22, 63) that denature the enzyme $[39,40]$.

\section{Synthesis of Asp and Glu acid oligomers}

The synthesis of di-carboxylic amino acids Asp and Glu oligomers was also evaluated in three-phase and monophasic systems. The synthesized ester of Asp and Glu (Figures 4A and 4B) was used as

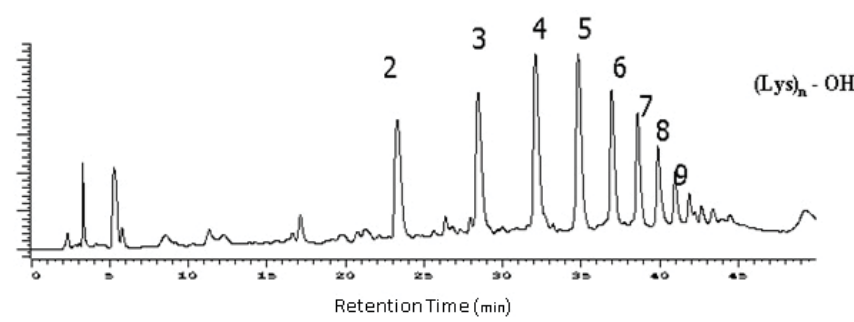

Figure 2: RPLC separation of Lys oligomers synthesized in three-phase system with L-Cysteine as the anti-oxidant. Separation was achieved in a RPLC C-18 column with HSA as the ion-pairing agent in the mobile phase using a mobile phase gradient mentioned in Figure 1. The separation shows the presence of peaks corresponding to oligomers of Lysine. 
the substrate for oligomerization of Asp and Glu in three-phase and monophasic system in the presence of L-Cysteine and mercaptoethanol. The RPLC chromatogram of the residual monomers left in the Asp oligomers synthesized in three-phase system with L-Cysteine and mercaptoethanol for $24 \mathrm{~h}$ incubation is shown in Figures 5A and 5B.

The chromatogram shows a clear separation of Asp, Asp monoester and Asp diester. The percent conversion of the reaction was calculated from the residual monomer left in the reaction mixture as mentioned above.

The separation of residual monomers in the Glu oligomerization product obtained from three-phase system for $24 \mathrm{~h}$ incubation is shown in Figures 6A and 6B.

Conversion was close to $80 \%$ in the presence of L-Cysteine while it was close to $40 \%$ in the presence of mercaptoethanol. Similar conversion was obtained for Glu oligomerization.

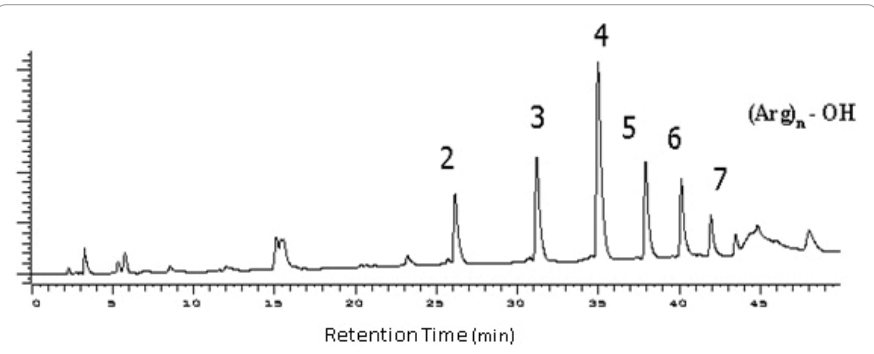

Figure 3: RPLC separation of Arg oligomers synthesized in three-phase system with L-Cysteine as the anti-oxidant. Separation was achieved in a RPLC C-18 column with HSA as the ion-pairing agent in the mobile phase using a mobile phase gradient mentioned in Figure 1. The separation shows the presence of peaks corresponding to oligomers of Arginine.

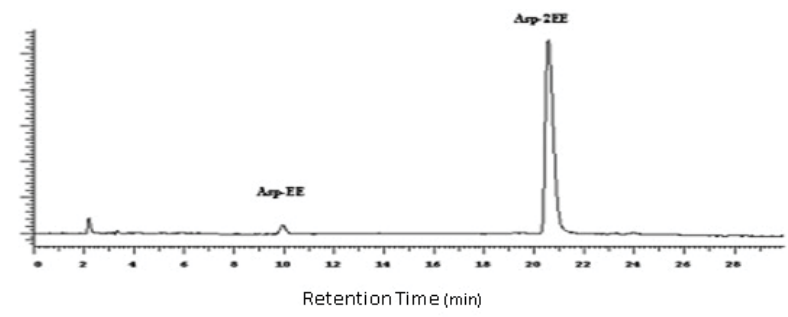

(A)

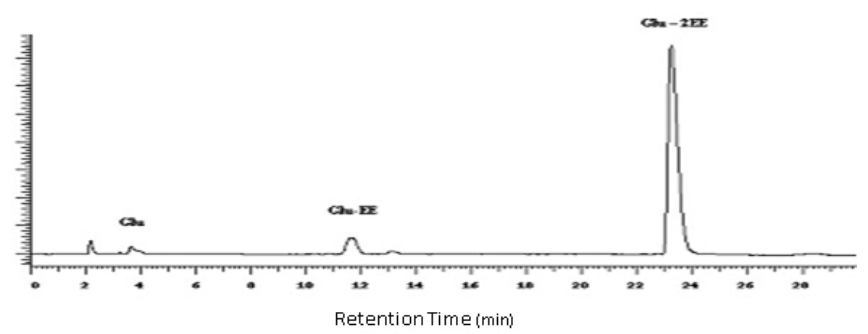

Figure 4: HPLC separation of acidic amino acid esters (A) Asp and (B) Glu synthesized with absolute ethanol. Separation was achieved with a RPC-18 column using a mobile phase gradient comprising of $100 \% \mathrm{~A}$ (Water $+10 \mathrm{mM}$ $\mathrm{HSA}+0.1 \%$ O-Phosphoric acid) to $60 \% \mathrm{~B}(50 \%$ acetonitrile $+10 \mathrm{mM} \mathrm{HSA}+0.1 \%$ O-Phosphoric acid) in 30 minutes. The chromatograms show the presence of two peaks eluting after the retention time of Asp and Glu corresponding to their mono and di-esters, respectively.

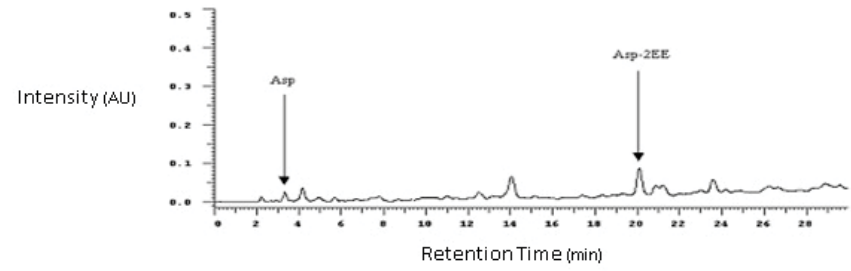

(A)

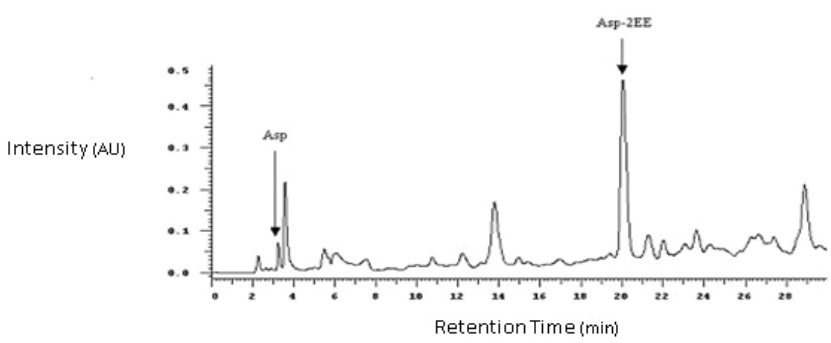

(B)

Figure 5: RPLC separation of residual monomers left in the Asp oligome product synthesized in three-phase system with (A) L-Cysteine and (B) 2-mercaptoethanol as anti-oxidant. Separation was achieved in a RPLC C-18 column with HSA as the ion-pairing agent in the mobile phase using a mobile phase gradient mentioned in Figure 4. The chromatograms show the clear separation of residual Asp ester and monomer.

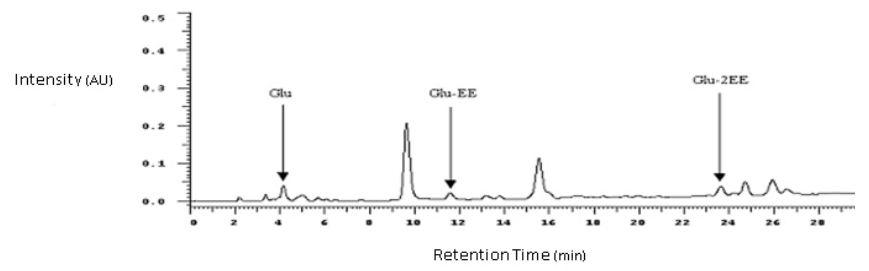

(A)

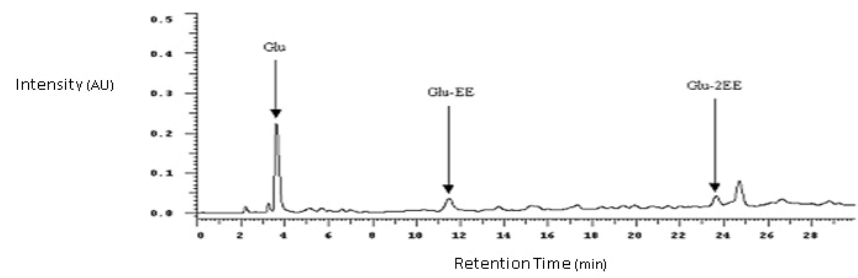

(B)

Figure 6: RPLC separation of residual monomers left in the Glu oligome product synthesized in three-phase system with (A) L-Cysteine and (B) 2-mercaptoethanol as anti-oxidant. Separation was achieved in a RPLC C-18 column with HSA as the ion-pairing agent in the mobile phase using a mobile phase gradient mentioned in Figure 4 . The chromatograms show the clear separation of residual Glu ester and monomer.

The ESI (+)-MS analysis of the synthesized oligomer product was carried out to determine the product distribution in the oligomer chain. Figure 7A shows the ESI-MS spectrum of Asp oligomer synthesized in three-phase system with L-cysteine as the anti-oxidant, for a $24 \mathrm{~h}$ incubation period. The spectrum consists of a series of peaks appearing at $\mathrm{m} / \mathrm{z} 305,448,591,734,877$ and 1020 . These peaks appear at a mass difference of $143 \mathrm{amu}$ corresponding to an Asp residue backbone with an intact ester group. These peaks correspond to 2 to 7 residues of Asp. A simple Asp dimer is shown in Figure 8. 

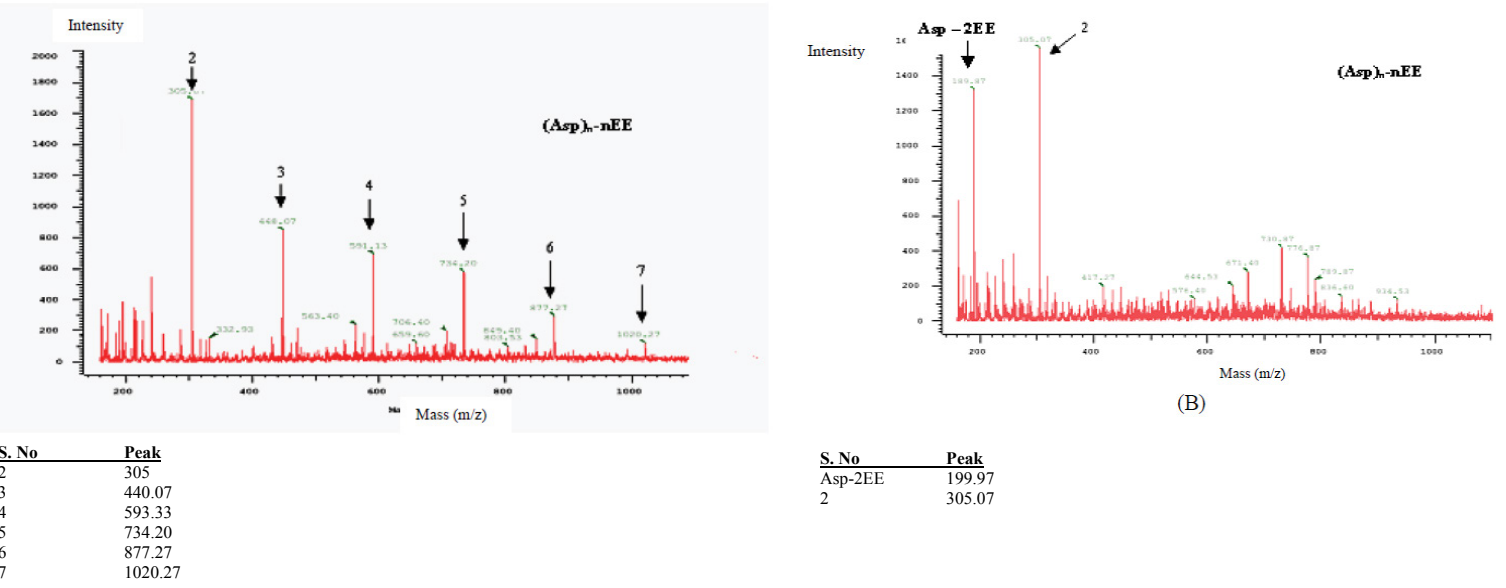

Figure 7: Direct injection ESI (+)-MS spectra of Asp oligomers synthesized with (A) L-Cysteine and (B) 2-mercaptoethanol as the anti-oxidant in three-phase system The spectrum shows the presence of series of peaks corresponding to oligomers of Asp.

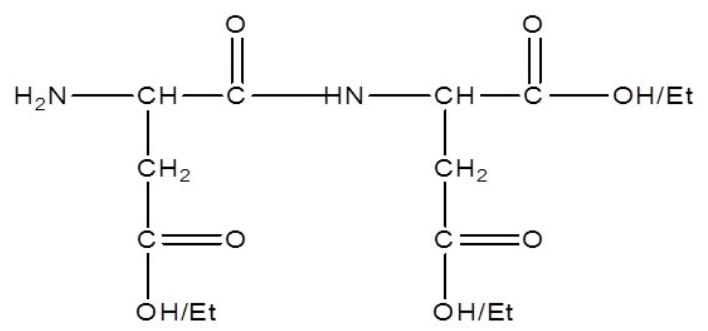

Figure 8: Structure of an Asp dimer indicating the possible sites for the presence of intact ester groups. Both the $\alpha$ - carboxyl group and the side chain carboxyl group can have intact ester moiety.

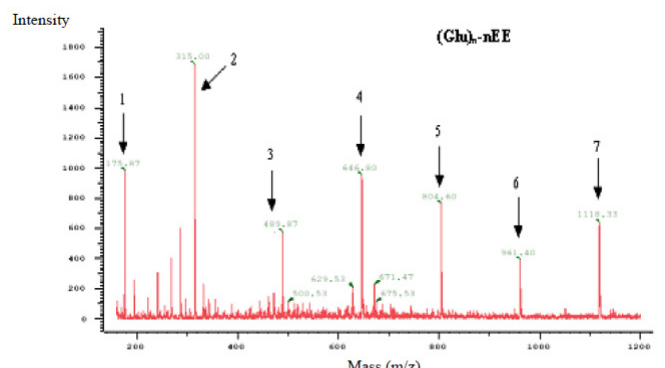

(A)

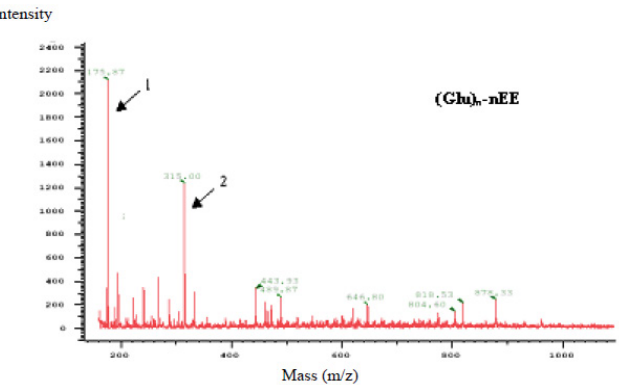

(B)
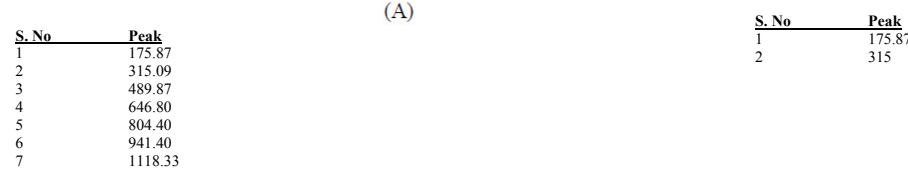
spectrum shows the presence of peaks corresponding to oligomers of Glu.

There are three possible sites for the presence of ester intact residues. The oligomer residues of Lys, Met, Arg and other amino acids obtained in a three-phase system typically has the $\alpha$ - carbon in a free acid form. This coupled with the dominant presence of diester in the starting substrate is an indication of the presence of intact ester residue in the side chain carboxylic acid group. When mercaptoethanol was used as the anti-oxidant in three-phase system, the mass spectrum of Asp oligomers showed a product consisting of only Asp dimer (Figure $7 \mathrm{~B})$. Oligomerization is more complete in the presence of L-Cysteine than in the presence of mercaptoethanol. Only the dimer is formed in mercaptoethanol whereas higher oligomers are formed in L-Cysteine.
The ESI - MS spectrum of Glu oligomers synthesized with L-Cysteine and mercaptoethanol in a three-phase system, for $24 \mathrm{~h}$ incubation is shown in Figures 9A and 9B.

Figure 9A consists of series of peaks appearing at $\mathrm{m} / \mathrm{z} 490,647,804$, 961 and 1118 with 157 amu difference indicated the formation of Glu oligomer products with residue numbers (n) 3, 4, 5, 6 and 7 respectively. The mass difference of $157 \mathrm{amu}$ corresponds to the addition of Glu backbone with intact ester residue. The ion at $\mathrm{m} / \mathrm{z} 316$ corresponds to a fragment ion of Glu dimer arising from the loss of ammonia as a neutral from the actual dimer ion. Only Glu dimer is formed in the presence of mercaptoethanol as the anti-oxidant (Figure 9B). 


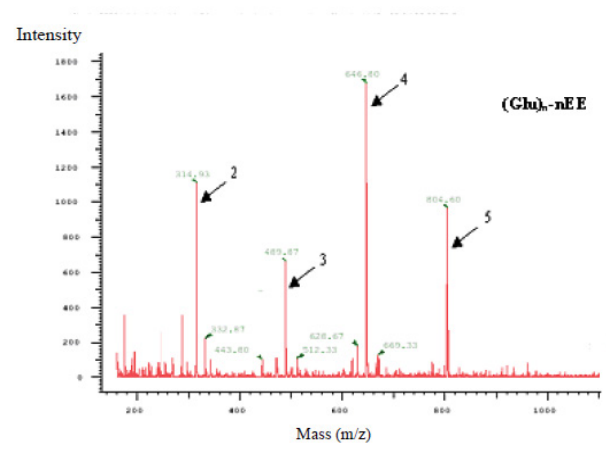

(A)

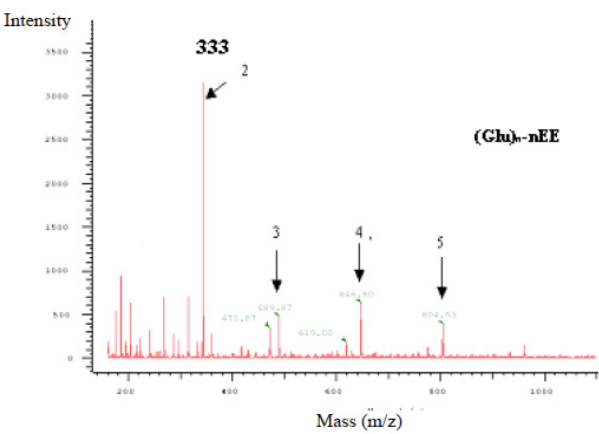

(B)
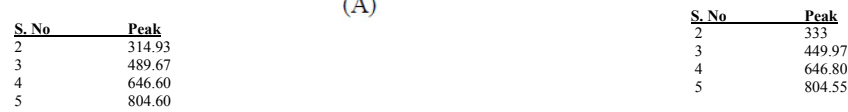

Figure 10: Direct injection ESI (+)-MS spectra of Glu oligomers synthesized with (A) L-Cysteine and (B) 2-mercaptoethanol as anti-oxidant in monophasic solvent system comprising of $90 \%$ acetonitrile/ $10 \%$ water. The spectrum consists of a series of peaks corresponding to Glu oligomers.

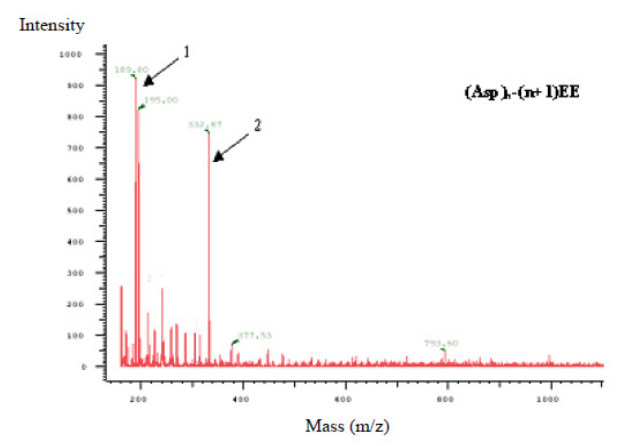

(A)

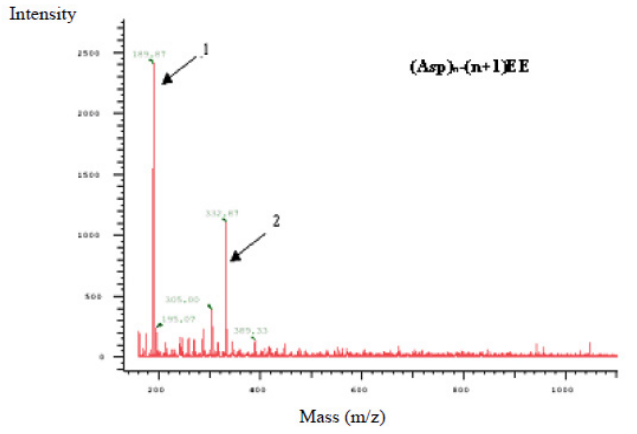

(B)
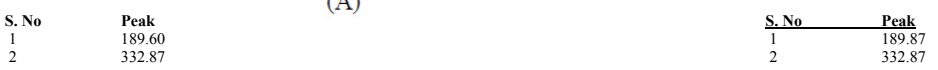

Figure 11: Direct injection ESI (+)-MS spectra of Asp oligomers synthesized with (A) L-Cysteine and (B) 2-mercaptoethanol as anti-oxidant in monophasic solvent system comprising of $90 \%$ acetonitrile/ $10 \%$ water. The spectrum shows the presence of only Asp dimer.

Asp and Glu oligomerization was also carried out in a monophasic system (acetonitrile/water (9:1)). The equilibrium was shifted towards peptide synthesis by the precipitation of the formed Asp and Glu oligomers from the reaction medium because of the presence of very little water. The percent conversion was calculated based on the residual amount of monomer left in the supernatant of the reaction mixture. The conversion was close to $80 \%$ for Glu oligomerization in the presence of L-Cysteine while it was only $50 \%$ in the presence of mercaptoethanol (Table 1), for a $24 \mathrm{~h}$ incubation of the reaction mixture. The ESI (+)-MS spectrum of the precipitated Glu oligomers synthesized in acetonitrile/ water system in the presence of L-Cysteine, for a $24 \mathrm{~h}$ incubation is shown in Figure 10A. The product profile though close to three-phase system, shows only the formation up to pentamer. The tetramer and pentamer were the dominant products. As the product precipitates out, the solubility of the higher oligomer residues decrease thereby reducing their availability as substrates for further oligomerization. This resulted in the formation of shorter chain oligopeptides in monophasic solvent system when compared to a three-phase system. The ion appearing at $\mathrm{m} / \mathrm{z} 316$ corresponds to the ion resulting from the fragmentation of Glu dimer in the MS with the loss of ammonia as a neutral. The precipitated product mainly comprised of an ion appearing at $\mathrm{m} / \mathrm{z} 333$ corresponding to a Glu dimer residue with two intact ester groups when mercaptoethanol was used as the reducing agent (Figure 10B).
The percent conversion for the Asp oligomerization reaction, based on the amount of monomer left in the supernatant in an acetonitrile/ water (9:1) solvent system with L-Cysteine as anti-oxidant for a $24 \mathrm{~h}$ incubation was only $45 \%$ (Table 1 ).

The reduction in oligomerization efficiency in case of Asp is due to the highly polar nature of Asp that results in the chemical hydrolysis of the synthesized oligomers even when only $10 \%$ water is present. The percent conversion dropped to $35 \%$ when mercaptoethanol was used as the reducing agent (Table 1). The ESI-MS analysis of the precipitated product synthesized in the presence of L-Cysteine and mercaptoethanol in a $10 \%$ water/ACN mixture for a $24 \mathrm{~h}$ incubation (Figures $11 \mathrm{~A}$ and 11B) revealed only the presence of a dimer with three intact ester groups appearing at $\mathrm{m} / \mathrm{z} 333$.

In order to increase the percent oligomerization of Asp, the solvent composition was changed to acetonitrile (95\%)/water (5\%). The RPLC separation of the supernatant from the reaction product in the presence of L-Cysteine, for a $24 \mathrm{~h}$ incubation (Figure 12) shows that the percent conversion of monomer was $\sim 80 \%$. The percent conversion in the presence of mercaptoethanol was only $35 \%$ (Table 1 ).

When the precipitated Asp oligomers synthesized in the presence of L-Cysteine was analyzed using ESI (+) - MS, the spectrum shows the presence of peaks appearing at $\mathrm{m} / \mathrm{z} 305,448,591,734,877$ corresponding 


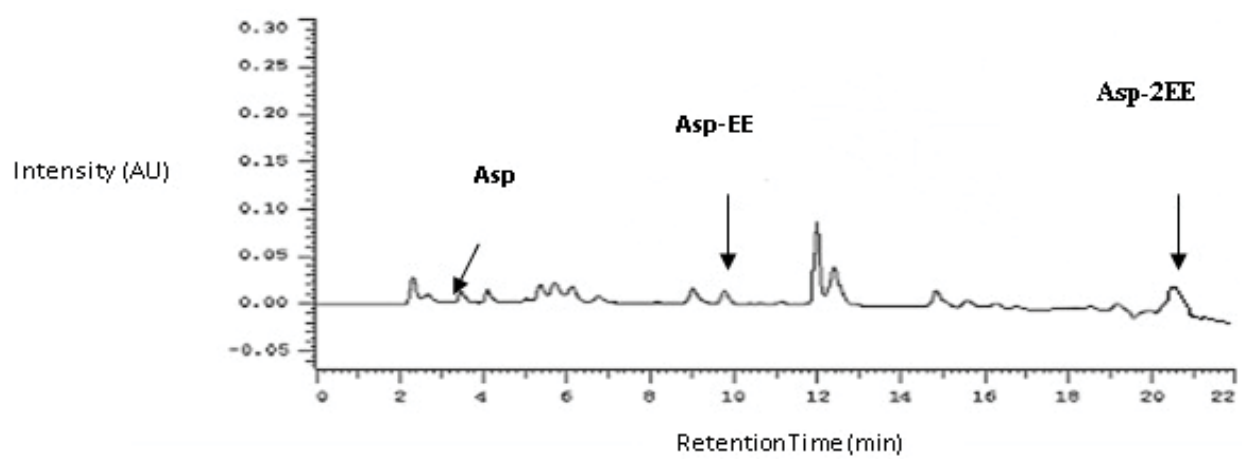

Figure 12: RPLC separation of residual monomers left in the Asp oligomer product synthesized in monophasic system with $95 \%$ acetonitrile and $5 \%$ water in the presence of L-Cysteine as an anti-oxidant for papain stabilization. Separation was achieved in a RPLC C-18 column with HSA as the ion-pairing agent in the mobile phase using a mobile phase gradient mentioned in Figure 4. The chromatogram shows the clear separation of residual Asp ester and monomer.
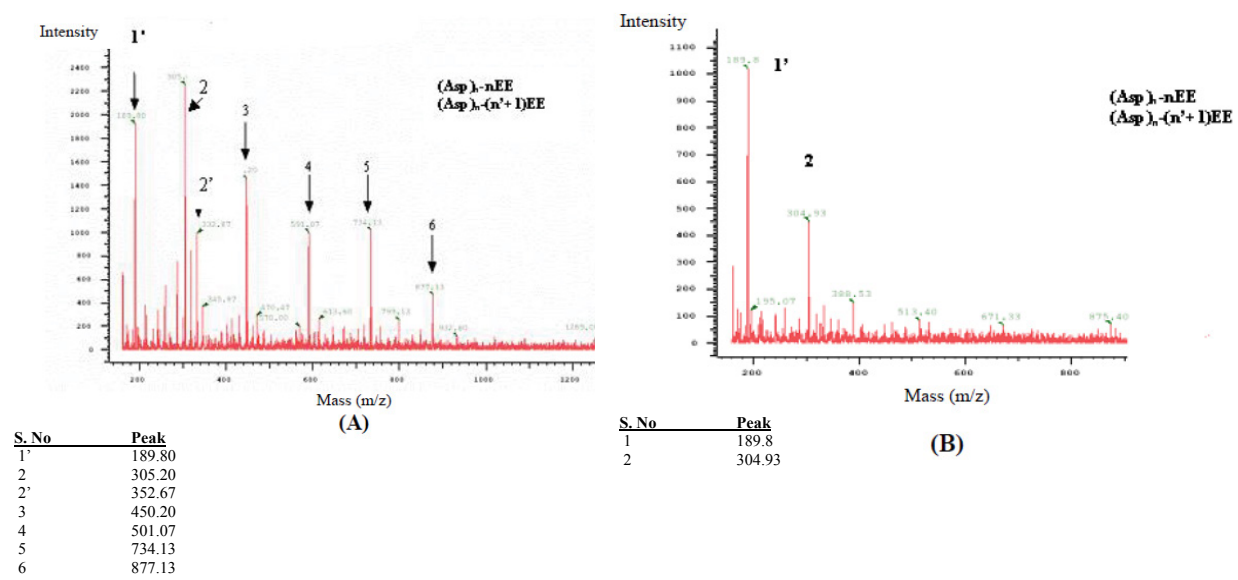

Figure 13: Direct injection ESI (+)-MS spectra of Asp oligomers synthesized with (A) L-Cysteine and (B) 2-mercaptoethanol as anti-oxidant in monophasic solvent system comprising of $95 \%$ acetonitrile/ $5 \%$ water. The top spectrum consists of a series of peaks corresponding to oligomers of Asp, while the bottom spectrum shows only the formation of Asp dimer.

to Asp oligomer residues ranging from 2 to 6 respectively (Figure 13A). Asp dimer dominated the product synthesized under the same solvent composition in the presence of mercaptoethanol (Figure 13B). The dimer in this case had only two ester intact residues.

Table 1 is a summation of results obtained for different amino acids with different anti-oxidants and also under anoxic conditions. The higher amounts of residual monomers left in the reaction medium incubated under anoxic conditions and in the absence of anti-oxidants show their importance in maintaining the activity of thiol proteases in reaction medium. The anoxic conditions used are not sufficient to maintain the cysteine residue of the active site in its reduced form (-SH) and it oxidizes and forms a disulfide bridge with another cysteine residue. Rzychon and Chmiel [39] reported that the catalytic activity of Cysteine proteases is mainly dependent on the formation of a thiolate/ imidazolium pair resulting from the proton transfer between Cys-25 and His-159 residue. Hussain and Lowe [40] have reported the amino acid sequence of Papain. The sequence shows that the Cys residues (Cys $-22,63)$ that are adjacent to the active site Cys- 25 can form a disulfide bridge, thereby preventing the proton transfer to the Histidine group during the catalytic process. L-Cysteine served as an effective antioxidant for oligomerization reactions in three-phase and monophasic solvent systems. Oligomers of Lys, Arg, Asp and Glu were successfully synthesized in the presence of L-Cysteine. The oligomerization efficiency in the presence of L-Cysteine was close to $80 \%$ and there was no change in product distribution for basic amino acid oligomers when mercaptoethanol was replaced with L-Cysteine. The results obtained in this study compare well with results reported by Roy, et al. [27] and Rajesh, et al. [28] that have also been presented in Table 1. In the study conducted by Roy et al. in triphasic organic solvent system for the synthesis of tailored oligopeptides, the conversion was close to $80 \%$ in the presence of Mercaptoethanol for co-oligopeptides of Lys/ Arg with Methionine. Rajesh et al. has reported that conversion with L-Cysteine as anti-oxidant in an aqueous system was close to $75 \%$. This augurs well with the results obtained in the monophasic aqueous organic system evaluated in this study where the conversion was close to $80 \%$ with L-cysteine as an anti-oxidant and is also in-line with results published by our group earlier [41]. This is also the first study to report the synthesis of acidic amino acid oligomers in both triphasic and monophasic reaction system. In case of acidic amino acid oligomers L-Cysteine provided better conversion than mercaptoethanol. The elimination of mercaptoethanol by L-Cysteine will result in reduction of product toxicity. We believe that the protease catalysis system devoid of Mercaptoethanol presented in this study provides the best opportunity to synthesize oligopeptides of acidic and basic amino acid oligomers that would be amenable as supplements for animal nutrition.

\section{Conclusion}

We have shown that anti-oxidants in the reaction medium are 
Citation: Srinivasan S, Kapila S, Forciniti D, Nam P (2017) Efficacy of L-Cysteine as an Anti-Oxidant in Papain Catalyzed Synthesis of Oligopeptides in Organic Solvent System. Biochem Anal Biochem 6: 335. doi: 10.4172/2161-1009.1000335

needed for papain-catalyzed oligomerization of basic and acid amino acids. Our results show that L-Cysteine may be used efficiently to replace mercaptoethanol as the anti-oxidant. The elimination of mercaptoethanol from the reaction mixture would allow the use of the synthesized oligopeptides as dietary supplements.

\section{References}

1. Filippova IU, Lysogorskaya EN (2003) Modified proteases for peptide synthesis in organic media. Russ J Bioorgan Chem 29: 496-501.

2. Ferjancic A, Puigserver A, Gaertner H (1991) Papain catalyzed polymerization of amino acids in low water organic solvents. Biotechnol Lett 13: 161-166.

3. Lozano P, Iborra JL, Manjon A, Combes D (1992) One-Step synthesis of GlyGly-PheNH $\mathrm{H}_{2}$ from $\mathrm{N}$-unprotected amino acid derivatives by papain in one-phase liquid media. Biotechnol Lett 14: 933-936.

4. Clapes P, Torres JL, Aldercreutz P (1995) Enzymatic peptide synthesis in low water content systems: Preparative enzymatic synthesis of [Leu]- and [Met] Enkephalin derivatives. Bioorganic and Medicinal Chemistry 3: 245-255.

5. Bacheva AV, Baibak OV, Belyaeva AV, Lysogorskaya EN, Oksenoit ES, et al. (2003) Native and modified subtilisin 72 as a catalyst for peptide synthesis in media with low water content. Russian Journal of Bioorganic Chemistry 29 502-508.

6. Rawlings ND, Barrett AJ (1994) Families of cysteine peptidases. Methods in Enzymology 244: 461-486.

7. Harrison MJ, Burton NA, Hillier IH (1997) Catalytic mechanism of the enzyme papain: Predictions with a hybrid quantum mechanical/molecular mechanical potential. J Am Chem Soc 119: 12285-12291.

8. Julia JD (1983) Utilization of supplemental methionine sources by primary cultures of chick hepatocytes. J Nutr 113: 2116-2123.

9. Younge BA, Murphy JJ, Rath M, Sloan BK (2001) Effect of dietary absorbable methionine and lysine concentrations on milk production and composition of dairy cows offered grass-silage based diets. Irish J. of Agri. and Food Res 40

10. Polan CE, Cummins KA, Sniffen CJ, Muscato TV, Vicini JL, et al. (1991) Responses of dairy cows to supplemental rumen-protected forms of methionine and lysine. J Dairy Sci 74: 2997-3013.

11. Daniel B, Hubert FG, Antoine JP (1989) Poly-L-Lysine and multioligo (L-Methionyl) poly-L-Lysine as nutritional sources of essential amino acids. J Agric Food Chem 37: 873-877.

12. IUPAC-IUBMB Joint Commission on Biochemical Nomenclature (1987) Nomenclature and Symbolism for Amino Acids and Peptides. Recommendations on Organic \& Biochemical Nomenclature, Symbols \& Terminology, etc. Waveland Press.

13. Wagenmakers AJ (1998) Protein and amino acid metabolism in human muscle. Adv Exp Med Biol 441: 307-319.

14. Wagenmakers AJ (1998) Muscle amino acid metabolism at rest and during exercise: role in human physiology and metabolism. Exer Sport Sci Rev 26 287-314

15. Wagenmakers AJ (1992) Amino acid metabolism, muscular fatigue and muscle wasting. Speculations on adaptations at high altitude. Int J Sports Med 1: 110113

16. Dienel GA, Cruz NF (2006) Astrocyte activation in working brain: Energy supplied by minor substrates. Neurochem Int 48: 586-95.

17. Okumoto $S$ (2005) Detection of glutamate release from neurons by genetically encoded surface-displayed FRET nano-sensors. Proceedings of the National Academy of Sciences U.S.A 102: 8740-8745.

18. Corrie JE (1993) Post-synaptic activation at the squid giant synapse by photolytic release of L-glutamate from a 'caged' L-glutamate. J Physiol 465 $1-8$

19. Bak LK, Schousboe A, Waagepetersen HS (2006) The glutamate/GABAglutamine cycle: aspects of transport, neurotransmitter homeostasis and ammonia transfer. J Neurochem 98: 641-653.

20. Pittenger C, Krystal JH, Coric V (2006) Glutamate-modulating drugs as novel pharmacotherapeutic agents in the treatment of obsessive-compulsive disorder. NeuroRx 3: 69-81.
21. Aso K, Uemura T, Shiokawa Y (1998) Protease-catalyzed synthesis of OligoL-Glutamic Acid from L-Glutamic Acid Diethyl Ester. Agric Biol Chem 52: 2443 2449 .

22. McKenna MC, Waagepetersen HS, Schousboe A, Sonnewald U (2006) Neuronal and astrocytic shuttle mechanisms for cytosolic-mitochondria transfer of reducing equivalents: Current evidence and pharmacological tools. Biochem. Pharmacol 71: 399-407.

23. Barron JT, Gu L, Parrillo JE (1998) Malate-aspartate shuttle, cytoplasmic $\mathrm{NADH}$ redox potential and energetics in vascular smooth muscle. J Mol Cell Cardiol 30: 1571-1579

24. Abel T, Knechtle B, Perret C, Eser P, Von Arx P, et al. (2005) Influence of chronic supplementation of arginine aspartate in endurance athletes on performance and substrate metabolism - A randomized, double-blind, placebo-controlled study. Int J Sports Med 26: 344-349.

25. Colombani PC, Bitzi R, Frey-Rindova P, Frey W, Arnold M, et al. (1999) Chronic arginine aspartate supplementation in runners reduces total plasma amino acid level at rest and during a marathon run. Eur J Nutr 38: 263-270.

26. Staedt U, Leweling H, Gladisch R, Kortsik C, Hagmuller E, et al. (1993) Effects of ornithine aspartate on plasma ammonia and plasma amino acids in patients with cirrhosis: A double-blind, randomized study using a four-fold crossove design. J Hepatol 19: 424-430.

27. Roy A (2003) Enantioselectivity of Hydroxy Methionine (HMB) incorporation in short chain peptide. PhD dissertation, University of Missouri-Rolla, USA

28. Rajesh MP (2003) Synthesis and characterization of hydroxy-acid capped cooligomers. PhD dissertation, University of Missouri-Rolla, USA.

29. http://www.worthington-biochem.com/PAP/default.html

30. http://ptcl.chem.ox.ac.uk/MSDS/ME/2-mercaptoethanol.html.

31. Anderson G, Luisi PL (1979) Papain-induced oligomerization of $\alpha$ - amino acid esters. Helevetica Chimica Acta 62: 488-496.

32. Jost R, Bambilla E, Monti JC (1980) Papain catalyzed oligomerization of alphaamino acids: Synthesis and characterization of water-insoluble oligomers of L-Methionine. Helevetica Chimica Acta 63: 375-384.

33. Nam P, Srinivasan S, Kapila S (2005) Synthesis and characterization of oligopeptides and hydroxy acid capped oligopeptides in mixed aqueous organic media. PITTCON. Orlando, Florida, USA.

34. Feliu JA, Mas C, Lopez-Santin J (1995) Studies on papain action in the synthesis of Gly-Phe in two-liquid-phase media. Enzyme microb. Technol 17 882-887.

35. Fergancic-Biagini A, Gaertner H, Puigserver A (1993) Papain catalyzed hydrolysis of and amino acid incorporation into BSA and Zein substrates in low water organic media. J Agric Food Chem 41: 1152-1157.

36. Kimura Y, Nakanishi K, Matsuno R (1990) Enzymatic synthesis of the precurso of Leu-enkephalin in water-immiscible organic solvent systems. Enzyme Microb Technol 12: 272-280.

37. Yun-Hun Y, Gui-Ling T, Guo-Wen X, Dong-Cheng T, Gang C, et al. (1998) Enzymatic synthesis of N-Protected Leu-enkephalin and some oligopeptides in organic solvents. Tetrahedron 54: 12585-12596.

38. Lee H, Fukushi H, Uchino M, Fukazawa $Y$ (1990) Papain catalyzed polymerization of L-a-Amino acid methyl esters with hydrophobic side chains Chemistry Express 5: 741-744.

39. Rzychon M, Chmiel D, Stec-Niemczyk J (2004) Modes of inhibition of cysteine proteases. Acta Biochimica Polonica 51: 861-873.

40. Hussain SS, Lowe G (1969) Completion of the amino acid sequence of papain Biochem J 114: 279-288.

41. Santhanakrishnan S, Shuben K, Paul N, Forciniti D (2017) Papain catalyzed oligomerization in monophasic aqueous organic media - Synthesis and characterization of neutral and polar amino acid oligomers. Biological Sciences (Unpublished manuscript). 